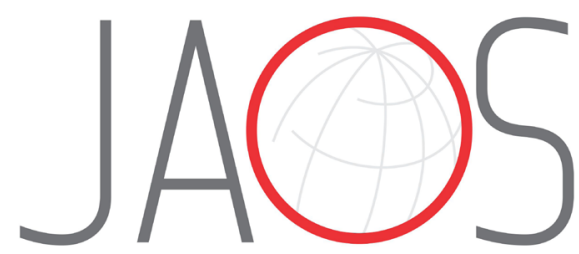
JOURNAL OF APPLIED ORAL SCIENCE

\title{
The association of self-reported awake bruxism with anxiety, depression, pain threshold at pressure, pain vigilance, and quality of life in patients undergoing orthodontic treatment
}

\section{Abstract}

Naila Aparecida Godoi MACHADO ${ }^{1,2}$

Yuri Martins COSTA $^{1,2}$ iD Henrique Muller QUEVEDO ${ }^{1,2}$ iD Juliana STUGINSKI-BARBOSA ${ }^{1,2}$ iD

Caio Martins VALLE ${ }^{1}$ iD Leonardo Rigoldi BONJARDIM ${ }^{2,3}$ (iD Daniela Gamba GARIB ${ }^{4}$ Paulo César Rodrigues CONTI ${ }^{1,2}$ iD
This study aimed to evaluate whether the presence of awake bruxism was associated with temporomandibular dysfunction symptoms, pain threshold at pressure, pain vigilance, oral health-related quality of life (OHRQoL), and anxiety and depression symptoms in patients undergoing orthodontic treatment. Methodology: This observational study followed patients who had started receiving orthodontic treatment for six months. The following variables were measured three times (at baseline, one month, and six months): pressure pain threshold (PPT) in the right and left masseter, anterior temporalis, and temporomandibular joint (TMJ), and right forearm; pain vigilance and awareness questionnaire; and shortened form of the oral health impact profile (OHIP-14). Anxiety and depression symptoms were measured using the Beck anxiety inventory and the Beck depression inventory, respectively. The patients were divided into two main groups according to the presence $(n=56)$ and absence $(n=58)$ of possible awake bruxism. The multi-way analysis of variance (ANOVA) was applied on the date $(p=0.050)$. Results: TMJ and/or muscle pain were not observed in both groups. Time, sex, age group, and awake bruxism did not affect the PPT in the masticatory muscles and pain vigilance $(p>0.050)$. However, the primary effect of awake bruxism was observed when anxiety (ANOVA: $F=8.61, p=0.004$ ) and depression (ANOVA: $F=6.48, p=0.012$ ) levels were higher and the OHRQOL was lower (ANOVA: $F=8.61, p=0.004$ ). Conclusion: The patients with self-reported awake bruxism undergoing an orthodontic treatment did not develop TMJ/masticatory muscle pain. The self-reported awake bruxism is associated with higher anxiety and depression levels and a poorer OHRQOL in patients during the orthodontic treatment.

Keywords: Orthodontics. Bruxism. Anxiety. Depression. Quality of life.
Submitted: July 22, 2019 Modification: November 6,2019 Accepted: November 13, 2019

Corresponding address: Naila Machado Departamento de Prótese e Periodontia - Faculdade de Odontologia de Bauru Universidade de São Paulo heiro Brisolla 9-75 - 17012 901 - Bauru - SP - Brasil. e-mail: naila_godoi@yahoo.com.br
${ }^{1}$ Universidade de São Paulo, Faculdade de Odontologia de Bauru, Departamento de Prótese e Periodontia, Bauru, São Paulo, Brasil.

2Universidade de São Paulo, Grupo de Dor Orofacial de Bauru, Bauru, São Paulo, Brasil. 3Universidade de São Paulo, Faculdade de Odontologia de Bauru, Departamento de Ciências Biológicas, Seção de Fisiologia da Cabeça e da Face, Bauru, São Paulo, Brasil.

${ }^{4}$ Universidade de São Paulo, Faculdade de Odontologia de Bauru, Departamento de Odontopediatria, Ortodontia e Saúde Coletiva, Seção de Ortodontia, Bauru, São Paulo, Brasil. 


\section{Introduction}

Bruxism is frequently implied as a source of microtrauma in the temporomandibular joints (TMJs) and in the mastication muscles. However, the evolution of new definitions and diagnostic criteria for bruxism has great repercussions for the possible relationship between bruxism and craniofacial pain. ${ }^{1}$ An international consensus recently defined bruxism as a repetitive jaw-muscle activity characterized by clenching or grinding of the teeth and/or by bracing or thrusting of the mandible, occurring within two distinct circadian manifestations: sleep and awake bruxism. ${ }^{1}$ Such specifications of the different motor activities and physiological brain states featuring the bruxism manifestations highlight the need to consider their possible different causes and clinical consequences.

Awake bruxism is a masticatory muscle activity during wakefulness that is characterized by repetitive or sustained tooth contact and/or by bracing or thrusting of the mandible, and is not a movement disorder in otherwise healthy individuals. ${ }^{1}$ Furthermore, the updated international consensus proposed a bruxism grading system to determine whether a certain bruxism assessment method actually offers a credible outcome. In addition, methods often used in the classification system for the bruxism diagnosis, such as self-report and clinical inspection, have been indicated as some of the only best leads to diagnose probable sleep or awake bruxism, and instrumental approaches are required for definitive bruxism assessments. ${ }^{1,2}$

Until now, the possible relationship between bruxism and symptoms of temporomandibular disorders is still controversial in the literature due to the complexity of etiology and diagnostic of both disorders. ${ }^{3-5}$ The hypothesis often discussed is the possible positive relationship between either awake or sleep bruxism and craniofacial pain is still a commonly held view in the clinical practice ${ }^{3-5}$, and sometimes even presented as a real and simple cause/effect relationship. In line with the perspective that painrelated temporomandibular disorders (TMD) must be envisaged within a biopsychosocial model of illness, and efforts to understand painful temporomandibular disorders along with other chronic pain conditions in a biopsychosocial context have been made..$^{5-8}$ This implies that the association between bruxism and painful temporomandibular disorders has become much more complex. ${ }^{5}$
Orthodontists should be aware of the presence of general and awake bruxism in particular and their possible implications during an orthodontic treatment, such as the excessive use of the jaw and possible association with dental structure (e.g., dental wear and restoration failures), TMJ, and masticatory muscle damage. ${ }^{9}$ Therefore, this study evaluates whether the presence of awake bruxism was associated with the occurrence of temporomandibular dysfunction symptoms, pain threshold at pressure, pain vigilance, oral health-related quality of life (OHRQoL), and anxiety and depression symptoms in patients undergoing orthodontic treatment. This study hypothesized a priori that patients with awake bruxism would present differences in deep pain sensitivity, pain vigilance, anxiety and depression symptoms, and OHRQoL, when compared with those without awake bruxism.

\section{Methodology}

\section{Sample and ethics}

The ethical approval was obtained from the Human Research Ethics Committee of the University of São Paulo, Brazil (CAAE - 09435812.4.0000.5417, June 2016). The participants were informed about the examination procedures and assured of the confidentiality of the collected information. Finally, all participants signed an informed consent form before their inclusion in this study.

The participants' recruitment was performed by selecting all the patients who started orthodontic treatment with fixed devices in the period from October 2013 to December 2015 in different specialization post-graduate programs in orthodontics. The study was conducted by three examiners who underwent training and calibration to ensure greater reliability for the data collected at different moments of the study. The minimum desired sample size for this study was calculated using an odds ratio (OR) of 2.0; the test power was $70.0 \%(\beta=.10)$, and the standard error was $5 \%(\alpha=.05)$.

This observational study followed patients who had started orthodontic treatment with fixed appliance for six months. The patients receiving treatment using removable appliances were not included in the sample. Initially, 162 patients were assessed for eligibility in several orthodontic specialization courses.

The inclusion criteria were the following: a) 
age between 13 and 60 years; b) absence of temporomandibular disorder (TMD) pain, which was assessed through the third question of the Research Diagnostic Criteria for Temporomandibular Disorders (RDC/TMD) questionnaire: "Have you felt pain on the face, in places such as the cheek (jaw) region, by the side of the head, in front of the ear or in the ear, in the last 4 weeks?". ${ }^{6}$ In addition, the exclusion criteria were the following: a) the presence of chronic orofacial pain, such as TMD or primary headache disorders; b) frequent cervical pain, fibromyalgia, and congenital or developmental disorders (e.g., aplasia, hypoplasia, hyperplasia, dysplasia, neoplasia). A detailed medical interview/anamnesis and a clinical examination were performed to fulfill the inclusion and exclusion criteria. No further tests were performed, such as imaging or blood tests.

\section{Variables}

The following variables were measured: a) pressure pain threshold (PPT); b) pain vigilance and awareness questionnaire (PVAQ); ${ }^{10,11} \mathrm{c}$ ) Beck anxiety inventory $(\mathrm{BAI}) ;{ }^{12}$ d) Beck depression inventory (BDI); ${ }^{12}$ and e) shortened form of the oral health impact profile $(\mathrm{OHIP}-14) \cdot{ }^{13}$

\section{PPT}

The PPT of right and left masseter, anterior temporalis and temporomandibular joint (TMJ), and right forearm was measured using a digital algometer (KRATOS ${ }^{\circledR}$, Cotia, São Paulo, Brazil). Measurements were obtained with the patient sitting comfortably in an upright position while the examiner pressed the $1-\mathrm{cm}^{2}$ tip at a rate of approximately $0.5 \mathrm{Kg} / \mathrm{cm}^{2} / \mathrm{s}$. The patients were instructed to press the stop button at the first painful sensation. It was highlighted that the purpose was to measure the minimal amount of pressure at the first perception of pain, and not the pain tolerance. The PPT was determined as the arithmetic mean of two measurements and the average of right and left sides were considered for the statistical analysis (see Statistics).

\section{PVAQ}

This self-report questionnaire comprises 16 items and is used to measure the attention to pain. The items are rated on a 6-point scale ranging from 0 (never) to 5 (always) and the final score is the sum of all individual items. The psychometric properties of the original version have shown acceptable values for retention (corrected item-total score correlations ranging from 0.36 to 0.76 ) and reliability (Cronbach's alpha $=0.92) \cdot{ }^{11,14}$

\section{BAI}

The anxiety symptoms were measured using the Beck Anxiety Inventory (BAI). This self-report questionnaire comprises 21 items and is used to measure the severity of anxiety. The items are rated on a 4-point scale, ranging from 0 (not at all) to 3 (severely), and the final score is the sum of all individual items. Accordingly, the BAI scores range from 0 to 63 , where the higher scores indicated higher anxiety symptoms. This study used the validated version for Portuguese, which showed good psychometrics (Cronbach's alpha $=0.81) .{ }^{12}$

\section{BDI}

The depression symptoms were measured using the Beck Anxiety Inventory (BAI). This self-report questionnaire comprises 21 items and is used to measure the severity of depression. The items are rated on a 4-point scale, ranging from 0 (not at all) to 3 (severely) and the final score is the sum of all individual items. Accordingly, the BDI-II scores range from 0 to 63, where the higher scores indicated higher depression symptoms. This study used the validated version for Brazilian Portuguese, which showed good psychometrics (Cronbach's alpha $=0.93) .{ }^{12}$

\section{OHIP-14}

This self-report questionnaire consists of 14 questions divided into seven domains: functional limitation, physical pain, psychological discomfort, physical disability, psychological disability, social disability, and handicap. The items are rated on a 5-point scale, ranging from 0 (never) to 4 (always), and the final score is the sum of all individual items. Accordingly, the OHIP-14 scores range from 0 to 56, where the higher scores indicated poor quality of life related to oral health. This study used the validated version for Brazilian Portuguese, which showed good psychometrics (intraclass coefficient $=0.87$ and Cronbach's alpha $=0.91) .{ }^{15}$

\section{Design}

After the enrollment, the patients were divided into two main groups according to the presence/absence of self-reported awake bruxism. This information was obtained from questions about daytime grinding 
or clenching of the teeth, which were adapted from the Oral Behavior Checklist (OBC); ${ }^{16}$ nonetheless, this study has not applied the full questionnaire. The patients' group with awake bruxism was composed according to the answers regularly, often, or always. The patients' group without awake bruxism was composed according to the following answers: "never", "sometimes".

For the age control, the sample was also divided into two groups according to the guidelines of the World Health Organization (WHO) for age groups: ${ }^{17}$ adolescents (aged 10 to 19 years inclusive) and adults (older than 19 years of age).

The variables were assessed at three time-points: T0 = baseline, i.e., just before the moment of installation of the fixed apparatus; $\mathrm{T} 1=$ one-month after the installation of the fixed apparatus; and T2 = six-months after the installation of the fixed apparatus.

\section{Statistics}

Quantitative data (age, PPT, PVAQ, BAI, BDI, and OHIP-14) were presented as means and standard deviation (SD) along with the sex distribution. Data normality was assessed using the Kolmogorov-Smirnov test. A log10 transformation was performed when the test results were significant, considering an alpha level of $5 \%(p<0.050)$.

A five-way Analysis of Variance (ANOVA) was applied as following: the repeated factor time (3 levels) and the factors site (4 levels), age group (2 levels), sex ( 2 levels), and awake bruxism (2 levels) were computed to compare the PPT values. In addition, a four-way ANOVA was applied as following: the repeated factor time ( 3 levels) and the factors age group (2 levels), sex (2 levels), and awake bruxism (2 levels) were computed to compare PVAQ, BAI,
BDI, and OHIP-14 values. When appropriate, post hoc analyses were performed using the Tukey's Honestly Statistical Difference (HSD). The significance level was set at $5 \%(p=0.050)$.

\section{Results}

One hundred and fourteen healthy participants fulfilled the eligibility criteria and were enrolled in this study. The mean age (SD) of the sample was 24.7 (11.1) and $52 \%$ of them were women. In addition, $49 \%$ reported awake bruxism, where the mean age (SD) was 27.3 (12.5) and $64 \%$ of them were women. The remainder $51 \%$ did not report awake bruxism, their mean age (SD) was 22.2 (9.1) and $41 \%$ of them were women. Tables 1 and 2 show the PPT values and the psychosocial outcomes throughout the follow-up according to the presence/absence of awake bruxism.

No main effects of time, sex, age group, and awake bruxism were observed on the PPT of masticatory muscles. However, site had a main effect (ANOVA: $F=733.64, p<0.001$ ), where the AT and the forearm showed higher thresholds than the masseter and the TMJ (Tukey: $p<0.001$ ). Also, there was an interaction between time and sex (ANOVA: $F=7.86, p<0.001$ ) where the women's thresholds at T0 were lower than T2 values (Tukey: $p=0.005$ ) and men's thresholds at T0 (Tukey: $p=0.032$ ).

There were no main effects of time, sex and age group, or awake bruxism on the pain vigilance $(p>0.050)$. Also, there were no main effects of time, sex, and age group on the anxiety and depression symptoms. However, there was a main effect of awake bruxism where its presence was related with higher anxiety (ANOVA: $F=8.61, p=0.004$ ) and depression

Table 1- Mean and standard deviation (SD) of the pressure pain threshold (PPT) of masseter, anterior temporalis, temporomandibular joint (TMJ), and right forearm throughout the study follow-up $(p<0.050)$

\begin{tabular}{clll}
\hline & Baseline & One-month & Six-months \\
\hline Awake Bruxism (n=56) & & & $2.81(0.93)$ \\
Masseter & $2.79(0.84)$ & $2.73(0.91)$ & $2.84(0.83)$ \\
Anterior Temporalis & $2.81(0.84)$ & $2.77(0.88)$ & $3.10(1.10)$ \\
TMJ & $3.07(1.07)$ & $3.09(1.14)$ & $5.36(1.49)$ \\
\hline Right forearm & $5.20(1.72)$ & $5.40(1.62)$ & $2.86(0.76)$ \\
W/o Awake Bruxism (n=58) & & & $2.80(0.74)$ \\
Masseter & $2.75(0.78)$ & $2.88(0.76)$ & $3.22(0.90)$ \\
\hline Anterior Temporalis & $2.83(0.72)$ & $2.87(0.72)$ & $5.27(1.20)$ \\
\hline TMJ & $3.07(0.92)$ & $3.21(0.92)$ & $5.29(1.27)$ \\
\hline Right forearm & $5.28(1.44)$ & &
\end{tabular}


Table 2- Mean and standard deviation (SD) of the pain vigilance and awareness questionnaire (PVAQ), beck anxiety inventory (BAI), beck depression inventory (BDI), and short-form of the oral health impact profile (OHIP-14) throughout the study follow-up ( $p=0.050)$

\begin{tabular}{cccc}
\hline & Baseline & One-month & Six-months \\
\hline Awake Bruxism (n=56) & & & $38.00(14.97)$ \\
PVAQ & $39.92(15.20)$ & $38.25(15.03)$ & $8.76(5.93)$ \\
BAI & $8.67(6.39)$ & $8.74(6.30)$ & $9.69(5.25)$ \\
BDI & $10.32(8.69)$ & $9.76(8.33)$ & $9.71(5.18)$ \\
\hline OHIP-14 & $11.78(6.18)$ & $10.16(5.15)$ & $33.79(15.39)$ \\
\hline W/o Awake Bruxism (n=58) & & & $6.10(7.39)$ \\
\hline PVAQ & $35.86(16.60)$ & $34.63(15.70)$ & $6.65(6.41)$ \\
\hline BAI & $5.51(5.18)$ & $5.68(7.46)$ & $8.13(5.83)$ \\
\hline BDI & $7.41(7.25)$ & $6.82(7.26)$ & $8.55(6.20)$ \\
\hline
\end{tabular}

(ANOVA: $F=6.48, p=0.012$ ) levels. Finally, although there were no main effects of time, sex, and age group on the ORHQoL ( $p>0.050)$, a worse ORHQoL was reported by patients with awake bruxism (ANOVA: $F=8.61, p=0.004$ ).

\section{Discussion}

Patients with self-reported awake bruxism undergoing orthodontic treatment did not develop $\mathrm{TMJ} /$ masticatory muscle pain. The exclusion of patients affected by previous TMJ pain might have biased the study sample. That might have influenced the composition of an artificially "healthier" sample, and, as a possible consequence, decrease the association "strength" between the presence awake bruxism and the pain-related symptoms.

This observational study evaluated the impact of awake bruxism on deep pain sensitivity, pain vigilance, anxiety and depression symptoms, and OHRQoL in patients during orthodontic treatment. The main results were: a) self-reported awake bruxism and six months of orthodontic treatment do not significantly influence the PPT of masticatory muscles in patients without signs and symptoms of TMD pain; b) higher anxiety and depression levels and a worse OHRQoL are found in patients who report awake bruxism regardless of the orthodontic treatment follow-up and age group.

The role of bruxism in the masticatory muscle sensitivity has been questioned. ${ }^{18,19}$ Although it is not possible to make definitive conclusions about the relationship between the presence of awake bruxism and orthodontic treatment, the results of this study showed that the orthodontic treatment and the presence of awake bruxism did not trigger the appearance of signs and symptoms of TMD pain, as no patients have complained of painful TMD after 6 months. These findings agree with the evidence that orthodontic treatment does not necessarily increase the risk of developing TMD. ${ }^{20}$

The results of no influence of neither the selfreported awake bruxism nor the orthodontic treatment on deep pain sensitivity also agree with experimental data, where healthy subjects demonstrated only low levels of pain and fatigue after an experimental tooth clenching paradigm, which was not associated with an altered release of serotonin, glutamate, lactate, or pyruvate. ${ }^{21}$ This evidence weakens the hypothesis that repetitive effort of the masticatory muscles motivated by bruxism episodes would be a risk factor for the development and maintenance of persistent muscle pain. The so called vicious-cycle theory, which was proposed to elucidate the relationship between bruxism episodes and the severity of pain, has not yet demonstrated convincing and definitive acceptance. ${ }^{22}$

Recently it was suggested that individuals with a high degree of oral parafunctional behavior, such as awake bruxism, have some increased occlusal sensitivity. ${ }^{23}$ Michelotti, et al. ${ }^{24}$ (2012) demonstrated that the effects of an experimental occlusal interference differ between individuals reporting a high or low frequency of wake-time oral parafunctions. The interference caused more occlusal discomfort in the high frequency of wake-time oral parafunctions group (HFP) than in the low frequency of wake-time oral parafunctions group and was associated with a significant increase in masticatory muscle pain and headache only in the HFP group. In this study, occlusal alterations resulting from the initial stages of orthodontic treatment associated with self-reported awake bruxism did not contribute to the appearance of symptoms of 
temporomandibular dysfunction during orthodontic treatment. Furthermore, the presence of awake bruxism was not associated with vigilance pain. New studies may be conducted evaluating the processes of attention in orthodontics, such as somatosensory amplification and hypervigilance. ${ }^{24}$ Clinical experience suggests that individuals with bodily hypervigilance also present with occlusal hypervigilance and continuously check their occlusion. ${ }^{25}$ The reaction to an occlusal alteration resulting from the initial stages of orthodontic treatment may be different in individuals with occlusal hypervigilance, which could justify some patients' difficulty in adapting to the orthodontic treatment.

The studies involving occlusion, orthodontics, and TMD up to the present moment have focused on the incidence and prevalence of dysfunctions in certain samples, seeking to establish the relative risk of this disease. However, since temporomandibular disorders are complex entities, the relationship between these entities should be seen in the pain models, inserting the biopsychosocial perspective in the evaluations. In the researchers' understanding, this is the first study to investigate the signs and symptoms of TMD in orthodontic patients, which also approaches the psychosocial aspects in the same sample.

Previous studies have highlighted the need to consider the psychological dimensions before and during the orthodontic treatment. ${ }^{26-29}$ The literature is scarce regarding information assessing anxiety and depression symptoms in patients during orthodontic treatment. Even though the results did not found a significant effect of the orthodontic treatment on psychosocial variables, the impact of awake bruxism on anxiety and depression symptoms and quality of life reinforce previous findings. They have showed an association with psychosocial factors, such as stress, disturbed personality, anxiety; psychopathological factors, such as smoking, consumption of alcohol and caffeine; and genetic factors. ${ }^{30,31}$ Traditional assumptions that peripheral factors such as occlusal discrepancies and deviations of the facial anatomy would be possible causes of bruxism have been discredited. ${ }^{32}$

Clinicians should be aware of the patients' psychological traits before starting orthodontic treatment, because these traits may be related to the presence of awake bruxism and to a significant impact on quality of life, compromising the patient's adaptation during orthodontic treatment. In addition, previous identification of patients with awake bruxism may have implications for the design of the treatment plans on measures, such as cognitive behavioral approaches that can be included and help patients understand their need for relaxed mastication muscles maintenance. This study has some limitations: a) the last international consensus defined and graded sleep and awake bruxism and determined that the selfreported diagnosis is classified as possible bruxism. In this study, the diagnosis of bruxism was performed without any clinical examination, which represents a lower grade of bruxism diagnosis uncertainty ("possible") according to the international bruxism group. ${ }^{1}$ However, it is also important to note that selfreported measures of oral parafunction have showed greater prognostic value than clinical assessment; ${ }^{33}$ b) the relatively large range between the follow-ups would have decreased the chance to detect transient changes, in particular the ones related to pain sensitivity, e.g., experimental data has showed that PPT is reduced after placing orthodontic separators. ${ }^{34}$

\section{Conclusion}

The self-reported awake bruxism is associated with higher anxiety and depression symptoms and poorer OHRQoL in patients during orthodontic treatment. On the other hand, short-term orthodontic treatment does not impact on deep pain sensitivity, pain vigilance, degree of anxiety and depression, and OHRQoL.

\section{Authors' Contribution}

Machado, N.A.G: Conception and design; Data acquisition; Data analysis and interpretation; Manuscript draft; Final approval and completed manuscript; Agreement to be accountable for all aspects of the work; Costa, Y.M.: Data analysis and interpretation; Manuscript draft; Final approval and completed manuscript; Agreement to be accountable for all aspects of the work; Quevedo, H.M.: Data acquisition; Revision of Intellectual content; Final approval and completed manuscript; Agreement to be accountable for all aspects of the work; StuginskiBarbosa, J.: Conception and design; Data analysis and interpretation; Final approval and completed manuscript; Agreement to be accountable for all aspects of the work; Valle, C.M.: Data acquisition; 
Data analysis and interpretation; Final approval and completed manuscript; Agreement to be accountable for all aspects of the work; Bonjardim, L.R.: Data analysis and interpretation; Revision of Intellectual content; Final approval and completed manuscript; Agreement to be accountable for all aspects of the work; Garib, D.G.: Data analysis and interpretation; revision of Intellectual content; Final approval and completed manuscript; Agreement to be accountable for all aspects of the work; Conti, P.C.: Conception and design; Data analysis and interpretation; Revision of Intellectual content; Final approval and completed manuscript; Agreement to be accountable for all aspects of the work.

\section{References}

1- Lobbezoo F, Ahlberg J, Raphael KG, Wetselaar P, Glaros A, Kato T, et al. International consensus on the assessment of bruxism: report of a work in progress. J Oral Rehabil. 2018;45(11):837-44. doi: 10.1111/joor. 12663

2- Lobbezoo F, Ahlberg J, Glaros AG, Kato T, Koyano K, Lavigne GJ, et al. Bruxism defined and graded: an international consensus. J Oral Rehabil. 2013;40(1):2-4. doi: 10.1111/joor.12011

3- Svensson P, Jadidi F, Arima T, Baad-Hansen L, Sessle BJ. Relationships between craniofacial pain and bruxism. J Oral Rehabil. 2008;35(7):524-47. doi: 10.1111/j.1365-2842.2008.01852.x

4- Huang GJ, LeResche L, Critchlow CW, Martin MD, Drangsholt MT. Risk factors for diagnostic subgroups of painful temporomandibular disorders (TMD). J Dent Res. 2002;81(4):284-8. doi: 10.1177/154405910208100412

5- Baad-Hansen L, Thymi M, Lobbezoo F, Svensson P. To what extent is bruxism associated with musculoskeletal signs and symptoms? A systematic review. J Oral Rehabil. 2019;46(9):845-61. doi: 10.1111/ joor.12821

6- Dworkin SF, LeResche L. Research diagnostic criteria for temporomandibular disorders: review, criteria, examinations and specifications, critique. J Craniomandib Disord. 1992;6(4):301-55.

7- Suvinen TI, Reade PC, Kemppainen P, Kononen M, Dworkin SF. Review of aetiological concepts of temporomandibular pain disorders: towards a biopsychosocial model for integration of physical disorder factors with psychological and psychosocial illness impact factors. Eur J Pain. 2005;9(6):613-33. doi: 10.1016/j.ejpain.2005.01.012

8- Slade GD, Fillingim RB, Sanders AE, Bair E, Greenspan JD, Ohrbach R, et al. Summary of findings from the OPPERA prospective cohort study of incidence of first-onset temporomandibular disorder: implications and future directions. J Pain. 2013;14(12 Suppl):T116-24. doi: 10.1016/j. jpain.2013.09.010

9- Jimenez-Silva A, Peña-Duran C, Tobar-Reyes J, FrugoneZambra R. Sleep and awake bruxism in adults and its relationship with temporomandibular disorders: a systematic review from 2003 to 2014. Acta Odontol Scand. 2017;75(1):36-58. doi: $10.1080 / 00016357.2016 .1247465$

10- McCracken LM. "Attention" to pain in persons with chronic pain: a behavioral approach. Behav Ther. 1997;28:271-84.
11- Sampaio Bonafé FS, Marôco J, Duarte Bonini Campos JA. Crosscultural validation of the Brazilian Portuguese version of the Pain Vigilance and Awareness Questionnaire. J Oral Facial Pain Headache. 2017;32(2):e1-e12. doi: 10.11607/ofph.1853

12- Gomes-Oliveira MH, Gorenstein C, Lotufo F Neto, Andrade LH, Wang YP. Validation of the Brazilian Portuguese version of the Beck Depression Inventory-II in a community sample. Rev Bras Psiquiatr. 2012;34(4):389-94. doi: 10.1016/j.rbp.2012.03.005

13- Slade GD. Derivation and validation of a short-form oral health impact profile. Community Dent Oral Epidemiol. 1997;25(4):284-90. doi: 10.1111/j.1600-0528.1997.tb00941.x

14- McWilliams LA, Asmundson GJ. Assessing individual differences in attention to pain: psychometric properties of the Pain Vigilance and Awareness Questionnaire modified for a non-clinical pain sample. Pers Individ Dif. 2001;31(2):239-46. doi: 10.1016/S0191-8869(00)00132-X 15- Oliveira BH, Nadanovsky P. Psychometric properties of the Brazilian version of the Oral Health Impact Profile-short form. Community Dent Oral Epidemiol. 2005;33(4):307-14. doi: 10.1111/j.16000528.2005.00225.x

16- van der Meulen MJ, Lobbezoo F, Aartman IH, Naeije M. Validity of the Oral Behaviours Checklist: correlations between OBC scores and intensity of facial pain. J Oral Rehabil. 2014;41(2):115-21. doi: 10.1111/joor.12114

17- World Health Organization - WHO. Definition of key terms. Geneva: WHO; 2013.

18- Manfredini D, Lobbezoo F. Relationship between bruxism and temporomandibular disorders: a systematic review of literature from 1998 to 2008. Oral Surg Oral Med Oral Pathol Oral Radiol Endod. 2010;109(6):e26-50. doi: 10.1016/j.tripleo.2010.02.013

19- Conti PC, Stuginski-Barbosa J, Bonjardim LR, Soares S, Svensson P. Contingent electrical stimulation inhibits jaw muscle activity during sleep but not pain intensity or masticatory muscle pressure pain threshold in self-reported bruxers: a pilot study. Oral Surg Oral Med Oral Pathol Oral Radiol. 2014;117(1):45-52. doi: 10.1016/j. oooo.2013.08.015

20- Hirsch C. No Increased risk of temporomandibular disorders and bruxism in children and adolescents during orthodontic therapy. J Orofac Orthop. 2009;70:39-50. doi: 10.1007/s00056-009-0820-3

21- Dawson A, Ghafouri B, Gerdle B, List T, Svensson P, Ernberg M. Pain and intramuscular release of algesic substances in the masseter muscle after experimental tooth-clenching exercises in healthy subjects. J Orofac Pain. 2013;27(4):350-60. doi: 10.11607/jop.1170

22- Murray GM, Peck CC. Orofacial pain and jaw muscle activity: a new model. J Orofac Pain. 2007;21(4):263-78; discussion 279-88.

23- Bucci R, Koutris M, Lobbezoo F, Michelotti A. Occlusal sensitivity in individuals with different frequencies of oral parafunction. J Prosthet Dent. 2019;122(2);119-22. doi: 10.1016/j.prosdent.2018.10.006 24- Michelotti A, Cioffi I, Landino D, Galeone C, Farella M. Effects of experimental occlusal interferences in individuals reporting different levels of wake-time parafunctions. J Orofac Pain. 2012;26(3):168-75. 25- Chow JC, Cioffi I. Effects of trait anxiety, somatosensory amplification, and facial pain on self-reported oral behaviors. Clin Oral Investig. 2019;23(4):1653-61. doi: 10.1007/s00784-018-2600-1 26- Javidi $H$, Vettore $M$, Benson PE. Does orthodontic treatment before the age of 18 years improve oral health-related quality of life? A systematic review and meta-analysis. Am J Orthod Dentofacial Orthop. 2017;151(4):644-55. doi: 10.1016/j.ajodo.2016.12.011

27- O'Brien K, Wright J, Conboy F, Chadwick S, Connolly I, Cook P, et al. Effectiveness of early orthodontic treatment with the Twinblock appliance: a multicenter, randomized, controlled trial. Part 2: Psychosocial effects. Am J Orthod Dentofacial Orthop. 2003;124(5):48894; discussion 494-85. doi: 10.1016/S0889540603006425 
28- Topcuoglu T, Yildirim O, Birlik M, Sokucu O, Semiz M. The effect of orthodontic extraoral appliances on depression and the anxiety levels of patients and parents. Niger J Clin Pract. 2014;17(1):81-5. doi: 10.4103/1119-3077.122850

29- Costa AA, Serra-Negra JM, Bendo CB, Pordeus IA, Paiva SM. Impact of wearing fixed orthodontic appliances on quality of life among adolescents: case-control study. Angle Orthod. 2016;86(1):121-6. doi: 10.2319/100514716.1.

30- Manfredini D, Lobbezoo F. Role of psychosocial factors in the etiology of bruxism. J Orofac Pain. 2009;23(2):153-66.

31- Tavares LM, Silva Parente Macedo LC, Duarte CM, Goffredo GS Filho, Souza Tesch R. Cross-sectional study of anxiety symptoms and self-report of awake and sleep bruxism in female TMD patients. Cranio. 2016;34(6):378-81. doi: 10.1080/08869634.2016.1163806
32- Lavigne GJ, Khoury S, Abe S, Yamaguchi T, Raphael K. Bruxism physiology and pathology: an overview for clinicians. J Oral Rehabil. 2008;35(7):476-94. doi: 10.1111/j.1365-2842.2008.01881.x

33- Slade GD, Ohrbach R, Greenspan JD, Fillingim RB, Bair E, Sanders $A E$, et al. Painful temporomandibular disorder: decade of discovery from OPPERA studies. J Dent Res. 2016;95(10):1084-92. doi: 10.1177/0022034516653743

34- Michelotti A, Farella M, Martina R. Sensory and motor changes of the human jaw muscles during induced orthodontic pain. Eur J Orthod. 1999;21(4):397-404. doi: 10.1093/ejo/21.4.397 